•综述・

\title{
海洋保护区管理与保护成效评估的方法与进展
}

\author{
宋瑞玲 1,3 姚锦仙 ${ }^{1,2 *}$ 吴恺悦 ${ }^{1}$ 张晓川 ${ }^{1,3}$ 吕 植 ${ }^{1,3}$ 朱争光 ${ }^{4}$ 殷丽洁 1,3 \\ 1 (北京大学生命科学学院, 北京 100871) \\ 2 (北京大学海洋研究院, 北京 100871) \\ 3 (北京大学自然保护与社会发展研究中心, 北京 100871) \\ 4 (国家海洋环境监测中心, 辽宁大连 116023)
}

\begin{abstract}
摘要: 全球物种多样性的持续下降使得生物多样性保护面临巨大挑战, 海洋生物多样性的保护任务尤其艰巨。海 洋保护区是保护生物多样性的有效方式之一, 如何对其成效进行评估是当前研究热点。然而, 目前针对海洋保护 区的评估体系较少, 而且评估指标多侧重于管理成效。近年来随着全球生物多样性监测网络和数据库的建立, 以 及多种新技术(如遥感、声呐系统、卫星追踪、基因组学等)在海洋生物多样性监测中的应用, 使得从生态系统到 基因水平的多层次连续监测成为可能。基于此，建议未来我国海洋保护区成效评估应在充分利用新技术方法的基 础上, 加强长期科学监测, 建立并完善生物多样性监测数据库和信息共享机制, 发展跨学科的综合保护成效评估 体系, 加强基于生物多样性监测的保护成效评估。
\end{abstract}

关键词：海洋保护区; 保护成效; 生物多样性监测

\section{Evaluation of the effectiveness of marine protected areas: Methodologies and progress}

\author{
Ruiling Song ${ }^{1,3}$, Jinxian Yao ${ }^{1,2 *}$, Kaiyue Wu ${ }^{1}$, Xiaochuan Zhang ${ }^{1,3}$, Zhi Lü ${ }^{1,3}$, Zhengguang Zhu ${ }^{4}$, Lijie Yin ${ }^{1,3}$ \\ 1 School of Life Sciences, Peking University, Beijing 100871 \\ 2 Institute of Ocean Research, Peking University, Beijing 100871 \\ 3 Center for Nature and Society, Peking University, Beijing 100871 \\ 4 National Marine Environmental Monitoring Center, Dalian, Liaoning 116023
}

\begin{abstract}
The continued decline of global biodiversity presents a huge challenge for biodiversity conservation, especially for marine biodiversity conservation. As an effective way to protect biodiversity, evaluating the effectiveness of MPAs (Marine Protected Areas) is becoming a critical issue. However, only limited assessment methodologies were specially designed for MPAs so far. Moreover, evaluation indicators have mainly focused on management effectiveness. Recently, the establishment of global biodiversity monitoring networks and databases and the application of new technologies (including remote sensing, sonar system, satellite tracking, and genomics) have provided available data and information for quantified conservation effectiveness evaluations at multiple levels from ecosystems to genes. Future evaluation should be based on long-term scientific monitoring with the assistance of new technologies, promoting the establishment of the biodiversity monitoring database and information sharing, and developing integrated and interdisciplinary evaluation systems to evaluate conservation effectiveness.
\end{abstract}

Key words: marine protected area; conservation effectiveness; biodiversity monitoring

海洋作为地球上最大的生态系统, 具有极其丰 富的生物多样性, 海洋物种约占全球生物物种总数 的 $80 \%$ (Halpern, 2014)。随着人口快速增长, 海洋开 发力度加大, 海洋生物多样性受到越来越大的威胁,
其保护迫在眉睫。海洋保护区(Marine Protected Areas, MPA)是保护典型海洋生态系统和生物多样 性、拯救珍稀濒危物种的重要方式(Hockings et al, 2006)。虽然全球海洋保护区的数量和面积持续增长,

收稿日期: 2018-01-09; 接受日期: 2018-03-19

* 通讯作者 Author for correspondence. E-mail: jxyao@pku.edu.cn 
但仍一定程度存在重数量规模、轻质量效益的问 题。大多数现有海洋保护区因规划不完善或管理技 术缺乏, 监测管控力量弱等原因, 导致保护区形同 虚设(Halpern, 2014)。针对此现状, 许多国家、地区 和机构陆续开展了保护区成效评估的研究和实践。

保护区成效评估主要包括管理成效评估和保 护成效评估。管理成效评估即评估保护区在多大程 度上实现了预期的管理目标, 主要评价保护区设计 与规划结果、管理体制及过程的充分性与合理性、 保护区目标的达成度(Hockings et al, 2006)。保护成 效评估即评价保护区对其主要保护对象的保护效 果及其在维持生物多样性和保障生态系统服务功 能等方面的综合成效(王伟等, 2016)。通过海洋保护 区成效评估, 可以帮助管理者明确保护现状, 识别保 护空缺和存在的问题，从而提高保护行动的有效性。

我国的海洋保护起步相对较晚, 海洋生物多样 性保护与经济发展矛盾突出(曾江宁等, 2016; 王伟 等, 2016), 未来既需要建立更多新的海洋保护区, 也亟需评估现有海洋保护区的保护成效。在海洋保 护区数量和面积持续增加的背景下，本文将总结目 前评估海洋保护区管理成效和保护成效的研究进 展，以期为我国海洋保护区成效评估提供参考。

\section{近年来海洋保护区快速增加}

\section{1 全球海洋保护区的发展}

自20世纪60年代以来, 随着全球对生物多样性 保护的重视, 海洋生物多样性保护得以加强(曾江 宁, 2013) , 但早期发展缓慢。1962年IUCN提出“海 洋自然保护区” 的概念之后, 各国陆续建立了海洋 保护区。截至1970年, 27 个国家建立了118个海洋保 护区(Björklund, 1974), 1994年增至1,306个(Kelleher, 1996), 2003年全球海洋保护区总数达3,858个(UNEP, 2003)。2010年联合国《生物多样性公约》第10次缔 约方大会提出 “至2020年底将海域面积的 $10 \%$ 列为 保护区域”。这推动了海洋生物多样性保护的快速 发展, 海洋保护区的数量和面积持续增加(Thomas et al, 2014)。截止到2017年9月, 全球海洋保护区覆 盖了 2,300 万 $\mathrm{km}^{2}$ (约 $6.35 \%$ )的海洋面积。近年来, 随 着超大保护区的建设, 预计 2025年有望达成覆盖 $10 \%$ 海洋面积的目标(Lisa \& Danile, 2016)。

\section{2 我国海洋保护区的发展}

狭义上我国的海洋保护区体系分为两大类型,
即海洋自然保护区和海洋特别保护区，其中，海洋 特别保护区又包括海洋公园(曾江宁等, 2016)。自 1963年我国建立第一个海洋保护区蛇岛老铁山自 然保护区后，海洋保护区的发展停滞了近20年(叶 有华等, 2008), 直至20世纪80年代海洋保护区的面 积和数量又开始逐步增加(图1)。在2005年开始建立 海洋特别保护区，2011年开始建立海洋公园。自 1980年以来, 平均每年新增约 7 个海洋保护区, 覆 盖的海洋总面积约 14 万 $\mathrm{km}^{2}$, 约占我国海域面积的 4.6\% (曾江宁等, 2016)。

综上所述，经过半个多世纪的发展，全球(包括 我国)海洋保护区的数量和面积在持续增长, 但与 生物多样性快速降低的现状相比(Halpern, 2014)仍 显不足。如何提高海洋保护区的管理质量和保护成 效，有效发挥其在生物多样性保护中的作用，是海 洋保护区研究中的重要问题(Wu et al, 2017)。

\section{海洋保护区的管理成效评估}

\section{1 主要的海洋保护区管理成效评估体系比较}

相比于陆地生物多样性的保护, 海洋保护区成 效评估的研究和实践不足，发展滞后(IUCN，2012; 洪晓巧和方秦华, 2016)。最初用于保护区管理成效 评估的方法大多是针对陆地生物多样性保护的体 系，其中应用范围最广的是IUCN世界保护区委员 会(World Commission on Protected Areas, WCPA)提 出的保护区管理框架，以及由此衍生出的评估体系 如自然保护区管理快速评估和优先性确定方法 (Rapid Assessment and Prioritization of Protected Area Management, RAPPAM) (Ervin, 2003)、“保护区管 理成效跟踪工具”(Management Effectiveness Tracking Tool, METT) (Stolton et al, 2003)等。

早期海洋保护区管理成效评估也多沿用这些 陆地保护区评估体系。直至2004年世界银行和世界 自然基金会(WWF)在WCPA框架和METT的基础上, 发布了“海洋保护区打分卡”体系(World Bank, 2004), 针对海洋保护区调整了部分评估指标, 但大体仍与 METT类似，并未充分考虑海洋的特殊属性如开放 性、流通性等。与此同时, IUCN编写了《海洋保护 区管理成效评估指南》，增加了针对海洋特殊属性 的内容, 是目前全球最权威和最全面的海洋保护区 管理成效评估体系(Pomeroy et al, 2005)。

此外, 一些研究机构也针对其评估的海洋保护 


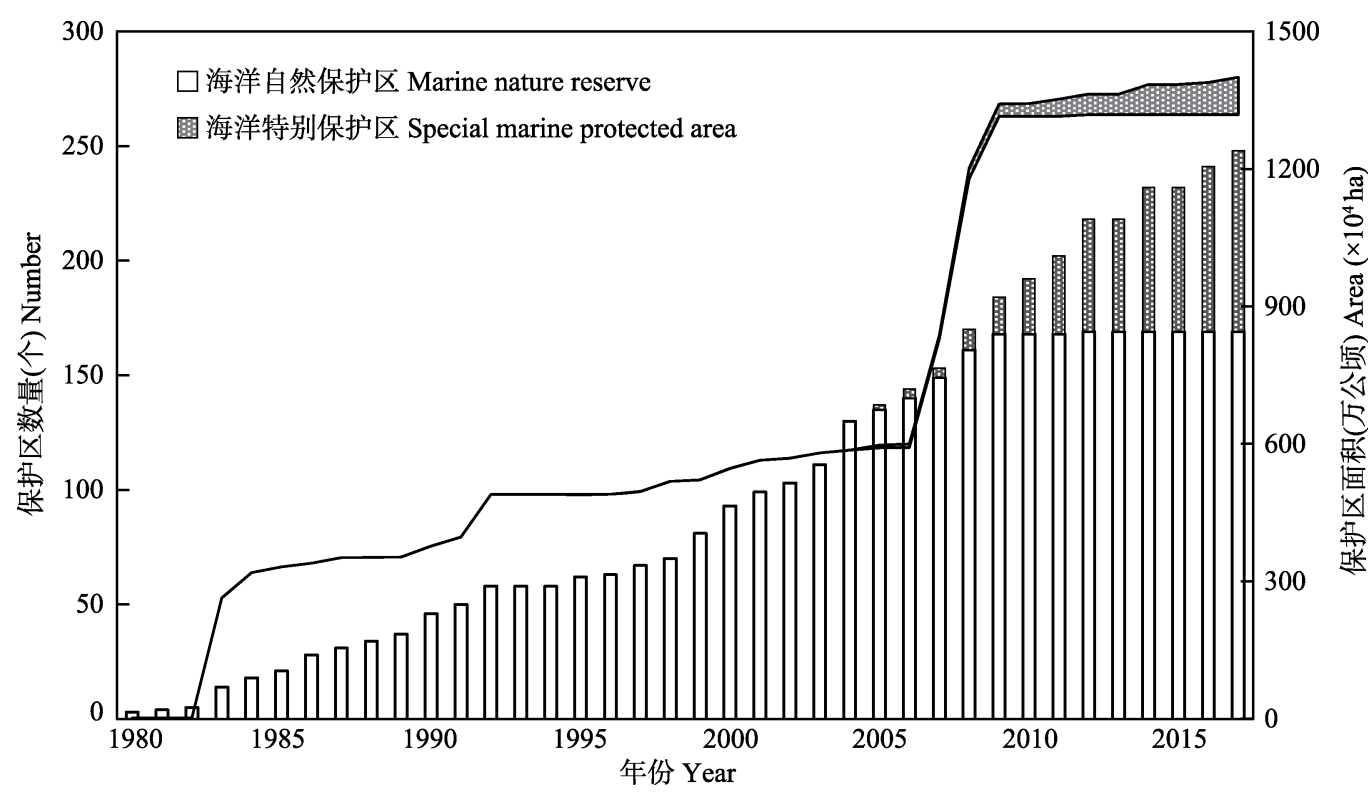

图1 自1980年以来中国海洋保护区的数量和面积持续增长(1980-2011年的数据来自曾江宁(2013), 2012-2017年的数据来自 国家海洋局网站)

Fig. 1 The number and area of marine protected areas in China have been growing since 1980. Data from 1980 to 2011 refer to Zeng (2013). Data from 2012 to 2017 refer to the website of State Oceanic Administration.

区特点, 综合了多种评估框架, 建立了新的评估体 系。如中美洲大堡礁项目 (Mesoamerican Barrier Reef Systems, MBRS)针对中美洲 4 个国家的珊瑚礁 系统制定了《中美洲海洋保护区管理成效快速评估 手册》(MBRS, 2004), 该体系在WCPA框架的基础 上, 增加了有针对性的指标如珊瑚礁和红树林盖 度、海草密度等。

表1列出了 4 种海洋保护区成效评估体系的特 征和应用情况对比。

\section{2 评估指标以管理和社会经济方面为主}

上述4种评估体系的指标均以管理和社会经济 方面为主(表1), 生物与环境方面的指标相对较少, 对于保护对象实际的状态与变化的评估比较薄弱。 METT和“海洋保护区打分卡”中缺少针对生物多样 性和生态系统的评估, 侧重经济投入和收益。IUCN 《海洋保护区管理成效评估指南》综合了社会经 济 管理和生物物理等3个方面的指标, 以前两者为 主(Pomeroy et al, 2005)。目前海洋保护区成效评估 的研究中应用最多的前 10 个评估指标中有 9 个是社 会经济和管理方面的, 包括利益相关者的参与度及 冲突, 当地人对海洋资源的认知、态度和利用, 捕 捞力量(fishing effort)的类型、水平和回报, 当地人 对法律法规的认知和服从等, 另一个是生物物理方 面“受人类影响消失或减少的区域” (Gallacher et al,
2016)。

除了应用已有的评估体系, 许多研究从社会经 济和管理的具体角度评估海洋保护区的成效。如关 注海洋保护区内利益相关者的冲突(Bennett \& Dearden, 2014; D’Anna et al, 2016), 经济发展如旅游业 的影响(Kaida \& Dang, 2016)、管理方式如建立海洋 保护区网络的成效(Horigue et al, 2014)、海洋保护区 内不同执法力度的保护效果(Guidetti et al, 2008)等。

这些评估体系和方法大多是定性调查, 与陆地 生物多样性保护成效评估情况类似(王伟等, 2016)。 主要通过文献调研和对不同利益相关方(如政府部 门、非政府组织、研究机构、当地社区、旅游者等) 的访谈和调查来进行分析。评估内容更关注管理过 程本身，依赖文献调研和管理者或专家的观点，而 对于保护生物多样性和维持生态系统服务功能方 面的评价相对不足。如果仅考虑管理成效的评估结 果，而对于保护对象的状况评估不够，导致生物多 样性仍处于快速下降状态，那么保护区的成效评估 有效性就值得商榷(权佳等, 2010)。因此在目前的保 护区成效评估体系里亟需增加基于生物多样性和 生态系统的评估指标。

\section{基于生物多样性监测的保护成交}

目前全球已建立了多种不同尺度和主题的生 
表1 主要海洋保护区管理成效评估体系比较

Table 1 Comparison of main methodologies for assessing management effectiveness of marine protected area

\begin{tabular}{|c|c|c|c|c|}
\hline & $\begin{array}{l}\text { METT } \\
\text { Management } \\
\text { Effectiveness } \\
\text { Tracking Tool } \\
\end{array}$ & $\begin{array}{l}\text { 海洋保护区打分卡 } \\
\text { Marine Tracking Tool }\end{array}$ & $\begin{array}{l}\text { 海洋保护区管理成效 } \\
\text { 评估指南 } \\
\text { How is your MPA doing }\end{array}$ & $\begin{array}{l}\text { 中美洲海洋保护区管理成效快速评估手册 } \\
\text { Manual for the Rapid Evaluation of Manage- } \\
\text { ment Effectiveness in Marine Protected Areas } \\
\text { of Mesoamerica }\end{array}$ \\
\hline 开发机构 & WWF/世界银行 & WWF/世界银行 & IUCN/WWF/NOAA & 中美洲大堡礁系统 \\
\hline Developer & WWF/World Bank & WWF/World Bank & & Mesoamerican Barrier Reef System \\
\hline $\begin{array}{l}\text { 评估人员 } \\
\text { Objects }\end{array}$ & $\begin{array}{l}\text { 保护区管理者、工作人员 } \\
\text { Managers and staff }\end{array}$ & $\begin{array}{l}\text { 保护区管理者、工作 } \\
\text { 人员、专家 } \\
\text { Managers, staff and } \\
\text { experts }\end{array}$ & \multicolumn{2}{|c|}{\begin{tabular}{|l} 
保护区管理者 、工作人员、专家、相关官员、社区居民 \\
Managers, staff, experts, relevant officials, and local communities
\end{tabular}} \\
\hline
\end{tabular}

人员专业要求 低 Low
低 Low
高 High
高 High

Professional

requirements

\begin{tabular}{|c|c|c|c|c|}
\hline $\begin{array}{l}\text { 人力成本 } \\
\text { Human cost }\end{array}$ & 低 Low & 低 Low & 高 High & 较低 Lower \\
\hline $\begin{array}{l}\text { 可重复性 } \\
\text { Repeatability }\end{array}$ & 好 Good & 好 Good & 不好 Bad & 较好 Better \\
\hline $\begin{array}{l}\text { 主观影响 } \\
\text { Effect of } \\
\text { subjectivity }\end{array}$ & 较小 Smaller & 较大 Larger & 较小 Smaller & 较小 Smaller \\
\hline $\begin{array}{l}\text { 针对性 } \\
\text { Marine-relevant }\end{array}$ & 无 None & 弱 Weak & 强 Strong & 强 Strong \\
\hline $\begin{array}{l}\text { 实践应用 } \\
\text { Application }\end{array}$ & $\begin{array}{l}\text { 至少用于 } 86 \text { 个国家的 } 1,150 \text { 个 } \\
\text { 保护区 } \\
\text { Applied in } 1,150 \text { protected } \\
\text { areas across over } 86 \text { countries }\end{array}$ & $\begin{array}{l}\text { 欧洲广泛应用 } \\
\text { Widely applied in } \\
\text { protected areas of } \\
\text { Europe }\end{array}$ & $\begin{array}{l}\text { 至少用于200多个保护 } \\
\text { 区 } \\
\text { Applied in over } 200 \\
\text { protected areas }\end{array}$ & $\begin{array}{l}\text { 中美洲大堡礁系统 } \\
\text { Applied in Mesoamerican Barrier Reef System }\end{array}$ \\
\hline $\begin{array}{l}\text { 应用建议 } \\
\text { Suggestion }\end{array}$ & $\begin{array}{l}\text { 可多次重复评估并监测变化 } \\
\text { Best for repeat evaluation and n }\end{array}$ & nitoring & $\begin{array}{l}\text { 指标综合全面 } \\
\text { Reference for } \\
\text { integrated indicators }\end{array}$ & $\begin{array}{l}\text { 适用于快速了解保护区管理和总体情况 } \\
\text { Best for a snapshot in performance } \\
\text { and general progress }\end{array}$ \\
\hline
\end{tabular}

$\begin{array}{lllll}\text { 指标数量 } & 30 & 34 & 42 & 47 \\ \text { Number of } & & & 42\end{array}$

Number of

indicators

共有指标

Common

indicators

立法与执法、管理计划、研究工作、人员管理与培训、经费预算、设备及维护、教育和意识培训

Legislation and law enforcement, management planning, research projects, staff management and training, budget, equipment and maintenance, education and awareness

保护区规章、目标、边界、资源普查信息和管理、当 当地利用海洋资源的活动和强度、依赖海洋资源的就业情况、服务 地居民收入、访客设施和收费

Regulations, objectives, boundary, resource

inventory, local income, visitor facilities

基础设施、环境与生物指标

Practices and intensity of local use of marine resources, employment in activities related to marine resources, state of service infrastructure, biophysical indicators

利益相关者的参与、与管理者的沟通、满意度, 环境教育、守法情况

Participation, communication with managers, satisfaction of stakeholders, environment education, law compliance

特有指标

Specific

$-$

保护区的设计、土地和水的使 保护区是否在更大的 对海洋资源的价值观、识别威胁、标牌、志愿者项目

indicators
用、所在社区的投入、商业旅 海岸管理体系中、产 居民健康、市场、科学 Threat recognition, signs/labels, and volunteer 游 品与服务、社区福利 知识传播、社区组织水 program

Protected area design, land and Integration of MPA in 平、历史遗迹

water use, local investment, and a larger coastal man- Values of marine re-

commercial tourism agement plan, prod- sources, local health,

ucts and services, and market, spread of scien-

community welfare tific knowledge, level of

community organiza-

tion, and historical sites 
物多样性监测网络(傅伯杰和刘宇, 2014)。比如与海 洋保护相关的有全球尺度的GEOSS (Global Earth Observation System of Systems)和国家尺度的澳大 利亚陆地生态系统研究网络(Terrestrial Ecosystem Research Network, TERN), 其中有“海岸带和海洋 生态系统的管理与保护”的监测内容。此外还有专 题性监测网络, 如湿地和海洋生态系统监测网络 (http://geobon.org/become-a-bon/thematic-bons/)。此 外，不同组织和机构还陆续建立了多个海洋生物多 样性有关的数据库和网站, 如全球最大的含位点数 据的在线开放性数据库海洋生物地理信息系统 (OBIS)、世界海洋生物物种目录(WoRMS), 以及针 对单一类群的鱼库(FishBase)、藻库(AlgaeBase)等 (邵广昭等, 2014)。

同时，随着新技术(如遥感、声呐、卫星追踪、 基因组学等)的发展和应用, 为海洋保护区内生物 多样性及其影响因素的监测和评估提供了新手段, 使得从生态系统到基因水平的多层次连续监测成 为可能, 进一步为海洋保护区的保护成效评估提供 了数据支持。

\section{1 遥感技术的应用}

随着遥感和地理信息系统等技术的普及, 遥感 数据被广泛用于海洋生态系统水平变化的监测 (Ouellette \& Getinet, 2016)。根据卫星遥感的不同时 空分辨率的产品和服务，可用于监测海岸地区的土 地利用及覆盖的变化以及海水水质、海底地形地 貌、水下动植物群及其他生物地理信息。目前卫星 遥感已被应用于一些海岸线及栖息地类型的变化 监测和研究。如Srivastava等(2015)应用印度卫星遥 感数据(IRS-1B IRS P6/RESOURCESAT-1)绘制了印 度喀奇湾红树林的盖度和密度分布及其变化, 发现 其主要受降雨和海平面上升影响。Wenger等(2016) 将2007-2013年的大堡礁海洋公园的珊瑚礁盖度和 存活率监测数据与MODIS卫星图像提取的河口洪 水事件和水质变化数据结合分析, 发现海洋保护区 不能有效保护珊瑚礁免受洪水的强烈干扰。我国相 关研究针对的海洋保护区有湄洲湾(赵宗泽等, 2013)、西门岛(李利红等, 2013)、黄河三角洲(侯学 会和李新华，2015)、双台河口(王丹桂等, 2015)等, 主要关注海岸线和沿海栖息地类型的变迁。

近年来, 近地遥感(如无人机)在资源环境监 测、生物多样性保护等领域的研究也日渐增加(An- derson \& Gaston, 2013)。比如用于海岸带大比例尺 地形测绘(邓才龙等, 2014)、海岛管理(鲍平勇等, 2016)、栖息地(如红树林)分类绘图(冯家莉等, 2015)、 入侵物种如互花米草(Spartina alterniflora)的监测 (李晓敏等, 2017)等。

\section{2 声学技术的应用}

近几十年来, 声学技术的发展为底栖生境调查 和生物多样性监测提供了先进的技术手段(Kang et al, 2011)。这方面的研究主要有近海底栖生境的识 别与分类和鱼类生物多样性的监测。

利用高分辨率、高精度的声呐系统数据, 结合 地理空间分析方法, 可以实现近海底栖生境的自动 分类识别与评估，比传统调查方法(如水下探摸)准 确度高、成本低(李东, 2017)。国外基于声呐数据进 行海底底质分类的研究较多, 涉及的底栖类型包括 海底泥沙与礁石、海草、海绵、珊瑚礁等。Huvenne 等(2016)在大西洋东北Darwin Mounds的深海冷水 珊瑚海洋保护区，用高分辨率的侧扫声呐系统评估 了禁渔8年后深海冷水珊瑚的恢复情况，分析保护 区的作用。Barberá等(2012)综合应用侧扫声呐系统、 箱式取样器、水下摄像机等多种调查手段, 在地中 海西部的Menorca Channel的海洋保护区内绘制了 50-100 m深处的栖息地类型，记录了 600多个物种， 识别出物种和功能多样性的热点区域和重点保护 对象。我国在这方面的应用研究较少。李东(2017) 用多波束声呐系统数据结合实地调查建立了一套 自动化分类方法，结合历史海图构建了山东烟台龙 口湾近 50 年的形态演变过程, 用于分析人类活动对 近海底栖生境的影响。

\section{3 卫星追踪技术的应用}

卫星追踪技术(satellite tracking technology)大 大推动了迁徙物种的监测与研究 (马克平, 2016), 包 括多种海洋洄游生物。通过追踪动物的活动轨迹, 可以了解动物对栖息地的时间和空间利用格局，识 别出洄游通道、受食地、繁殖地等关键利用区，从 而识别重点保护区，合理规划海洋保护区的空间， 评估石油等资源开发的环境影响，为设定禁渔时段 提供有针对性的建议等(Reynolds et al, 2017)。

应用卫星追踪技术可评估海洋保护区对物种 活动范围的覆盖程度, 目前已有一些研究。如 Patterson 等 (2016) 追踪了 1 种海狮 (Arctocephalus gazella)、2 种企我 (Eudyptes chrysolophus 和 
Aptenodytes patagonicus)、2 种信天翁 (Diomedea melanophrys和Phoebetria palpebrata)共5种178只个 体的栖息地利用，发现澳大利亚联邦海洋保护区只 覆盖了这些物种受食区域的不到20\%的面积。此外, 海洋保护区往往偏重于保护迁徙物种如海龟的繁 殖地，而忽略受食地和洄游通道(叶明涁等，2015; Zeh et al, 2016)。目前国外有大量研究应用卫星追踪 技术来获取多种海洋脊椎动物的活动轨迹和空间 利用，包括海龟(如Chelonia mydas, Thomson et al, 2017)、鲨鱼(如Rhincodon typus, Reynolds et al, 2017)、鲸(如Delphinapterus leucas, Hauser et al, 2017)、儒艮(Dugong dugon, Zeh et al, 2016)以及信 天翁和企鹅(Patterson et al, 2016)等。国内的研究主 要针对广东惠东港口国家级海洋保护区的绿海龟 (Chelonia mydas, 叶明涁等，2015)。研究发现，惠 东绿海龟主要途经广东、海南及台湾近海海域, 在 海南岛和日本琉球群岛之间洄游。

\section{4 基因组学技术的应用}

物种的快速准确鉴定是海洋生物多样性保护 的基础。海洋生物门类繁多, 有的门类(如浮游植物) 因个体小而外部形态特征不明显，有的门类(如鱼 类)在不同环境诱导下存在表型可塑性和趋同进化 现象，因而难以使用传统分类学方法来鉴定。相比 而言，分子鉴定手段快捷准确，成为海洋生物鉴定 的重要手段。比如高通量测量、DNA条形码等技术, 被广泛应用于生物多样性研究、动物食性分析、外 来入侵物种的监测等领域(Shaw et al, 2016)。

综上所述, 新技术的发展和应用使得定量地评 估不同水平的生物多样性的状况和变化趋势成为 可能(Addison et al, 2015)。应用遥感技术可从生态 系统层面评估海岸保护区内土地利用和覆盖类型 的变化, 评估人类活动或气候变化等对栖息地的影 响; 应用声呐系统数据可评估近海保护区底栖生境 的类别和变化; 通过卫星追踪技术获得海洋迁徙物 种的栖息地利用信息，可以识别关键区域，分析现 有海洋保护区的保护现状和空缺; 基因组学技术可 以快速鉴定海洋物种，有助于建立海洋保护区的生 物本底信息和监测物种多样性变化。

\section{4 我国海洋保护区成效评估研㲾}

我国的评估实践仍处于探索阶段。2004年国家 标准化管理委员会发布了《海洋自然保护区管理技
术规范》(GB/T 19571-2004)，其中包含《海洋自然 保护区管理质量评价方法》，是我国首个全国性的 针对海洋保护区管理成效的评估办法。该体系评估 主体是保护区工作人员，主要基于主观评价，缺乏 对保护区监测、生态效果以及利益相关者参与机制 的评估。2015年国家海洋局发布了《国家级海洋保 护区监督检查办法(试行)》，据此对国家级海洋保护 区的管理与保护进行监督检查。

目前应用上述体系的研究案例较少(洪晓巧和 方秦华，2016)，多为应用国际评估体系或在此基础 上开发的新体系。比如我国南部生物多样性项目 (SCCBD)基于METT体系评估了南鹿列岛国家级海 洋自然保护区的管理成效(曾江宁, 2013)。王在峰等 (2011)分析了江苏省海门市蚛岈山牡蚛礁海洋特别 保护区的海域生态系统现状和特点，构建了包含环 境、结构、稳定性方面的生态系统健康评价指标体 系。Wu等(2017)在WCPA框架的基础上，综合国内 外多种评估体系, 针对青岛胶州湾海洋特别保护区, 构建了一套“环境表现指标系统”(Environment Performance Indicator, EPI)。这些评估工作积极推动了 海洋保护区的建设和发展(王伟等, 2016)。

\section{结论和建议}

目前国际上已经开发的保护区管理成效评估 体系主要从管理过程和社会经济方面评估管理目 标的实现程度，存在一些限制因素：如常用的评估 方法大多是从WCPA基础上衍生而来，理论基础薄 弱; 保护区的生态、社会、经济等方面的评估结果 难以整合(洪晓巧和方秦华，2016); 由于基础监测 数据的缺乏而量化不足等。近年来随着全球生物多 样性监测网络及新技术的发展和应用，可以从生态 系统到基因层次进行连续监测，这为海洋保护区的 保护成效定量评估提供了可能的数据基础。

基于已有研究, 结合本项目组在一些代表性国 家级海洋自然保护区的实地考察(王吴, 2017), 可以 看到我国大部分海洋保护区受经费、人才等因素制 约, 科研力量薄弱, 缺乏较为全面的海洋生物多样 性本底数据，监测工作和体系尚不完善，信息化建 设不足，而且已有的保护成效评估主要为保护区工 作人员自评或依赖专家的定性打分。因此，对于未 来我国海洋保护区的建设和成效评估研究, 本文提 出以下建议。 
(1)加强保护成效评估的理论研究, 探索建立 和完善适用于我国的海洋保护评估体系。

(2)加强海洋保护区的立体监测, 建立持续的 科学的数据收集系统。目前我国在一些数据积累和 收集整理方面还存在较大缺口, 比如物种多样性本 底数据和重点监测物种的种群变化数据等。不同尺 度数据的连续性和准确性是保护成效评估结果有 效性的保障，未来需投入更多的资源，加强保护区 与科研机构的合作，充分利用新的监测技术和方法， 建立长期的、科学的、系统的监测体系, 为海洋保 护区成效评估提供数据基础。

(3)加强海洋保护区的信息化建设和信息共享 机制。为使监测信息更好地服务于保护成效评估, 需进一步加强监测网络和数据库的建设, 促进监测 信息的网络化和共享, 使科研人员及其他海洋保护 相关从业者可方便及时地获取信息。

(4)加强基于生物多样性监测的保护成效评估, 建立综合的评估体系。综合运用跨学科的方法, 在 注重管理评估的同时, 加强针对生物多样性的保护 成效评估, 实现定量的、客观的、可重复的保护成 效评估，从而识别影响保护成效的具体因素。

致谢：感谢山水自然保护中心和 WWF (中国)的资 金支持。感谢北京大学生命科学学院卜思涵和陈航 通协助收集和整理资料。

\section{参考文献}

Addison PF, Flander LB, Cook CN (2015) Are we missing the boat? Current uses of long-term biological monitoring data in the evaluation and management of marine protected areas. Journal of Environmental Management, 149, 148-156.

Anderson K, Gaston KJ (2013) Lightweight unmanned aerial vehicles will revolutionize spatial ecology. Frontiers in Ecology and the Environment, 11, 138-146.

Bao PY, Zhang Z, Xie JQ, Gao N, Cai ZG, Liu WW (2016) Application of GNSS precise control network rapid setting technology for UAV remote sensing monitoring in remote islands. Ocean Development and Management, 33(z2), 101-104. (in Chinese with English abstract) [鲍平勇, 张钊, 谢家颀, 高宁, 蔡志刚, 刘微微 (2016) 快速GNSS精密 布网在偏远海岛无人机遥感监测中的应用. 海洋开发与 管理, 33(z2), 101-104.]

Barberá C, Moranta J, Ordines F, Ramón M, Mesa AD, Díaz-Valdés M, Grau A, Massutí E (2012) Biodiversity and habitat mapping of Menorca Channel (western Mediterra- nean): Implications for conservation. Biodiversity and Conservation, 21, 701-728.

Bennett NJ, Dearden P (2014) Why local people do not support conservation: Community perceptions of marine protected area livelihood impacts, governance and management in Thailand. Marine Policy, 44, 107-116.

Björklund MI (1974) Achievements in marine conservation. I. Marine parks. Environmental Conservation, 1, 205-223.

D’Anna G, Fernandez TV, Pipitone C, Garofalo G, Badalamenti F (2016) Governance analysis in the Egadi Islands Marine Protected Area: A Mediterranean case study. Marine Policy, 71, 301-309.

Deng CL, Liu YX, Tian ZW, Ren J, Peng L (2014) Application of UAV remote sensing in the monitoring of islands and coastal zones. Coastal Engineering, 33(4), 41-48. (in Chinese with English abstract) [邓才龙, 刘炎雄, 田梓文, 任 军, 彭琳 (2014) 无人机遥感在海岛海岸带监测中的应 用研究. 海岸工程, 33(4), 41-48.]

Ervin J (2003) WWF: Rapid assessment and prioritization of protected area management (RAPPAM) methodology. https://doi.org/10.1641/0006-3568(2003)053[0833:RAOPA M]2.0.CO;2. (accessed on 2017-11-10)

Feng JL, Liu K, Zhu YH, Li Y, Liu L, Meng L (2015) Application of unmanned aerial vehicles to mangrove resources monitoring. Tropical Geography, 35(1), 35-42. (in Chinese with English abstract) [冯家莉, 刘凯, 朱远辉, 李勇, 柳 林, 蒙琳 (2015) 无人机遥感在红树林资源调查中的应 用. 热带地理, 35(1), 35-42.]

Fu BJ, Liu Y (2014) Global ecosystem observation and research programs: Evolution and insights for future development. Progress in Geography, 33, 893-902. (in Chinese with English abstract) [傅伯杰, 刘宇 (2014) 国际生态系 统观测研究计划及启示. 地理科学进展, 33, 893-902.]

Gallacher J, Simmonds N, Fellowes H, Brown N, Gill N, Clark W, Biggs C, Rodwell LD (2016) Evaluating the success of a marine protected area: A systematic review approach. Journal of Environmental Management, 183, 280-293.

Guidetti P, Milazzo M, Bussotti S, Molinari A, Murenu M, Pais A, Spano N, Balzano R, Agardy T, Boero F, Carrada G, Cattaneo-Vietti R, Cau A, Chemello R, Greco S, Manganaro A, Notarbartolo di Sciara G, Russo GF, Tunesi L (2008) Italian marine reserve effectiveness: Does enforcement matter? Biological Conservation, 141, 699-709.

Halpern BS (2014) Conservation: Making marine protected areas work. Nature, 506, 167-168.

Hauser DDW, Laidre KL, Stafford KM, Stern HL, Suydam RS, Richard PR (2017) Decadal shifts in autumn migration timing by Pacific Arctic beluga whales are related to delayed annual sea ice formation. Global Change Biology, 23, 2206-2217.

Hockings M, Stolton S, Leverington F, Dudley N, Courrau J (2006) Evaluating Effectiveness: A Framework for Assessing Management Effectiveness of Protected Areas, 2nd 
edn. IUCN and Cambridge, Switzerland and UK.

Hong XQ, Fang QH (2016) On the progress of management effectiveness evaluation systems for marine protected area. Ocean Development and Management, 33(2), 95-100. (in Chinese with English abstract) [洪晓巧, 方秦华 (2016) 海 洋保护区管理有效性评估体系研究进展. 海洋开发与管 理, 33(2), 95-100.]

Horigue V, Aliño PM, Pressey RL (2014) Evaluating management performance of marine protected area networks in the Philippines. Ocean and Coastal Management, 95(4), 11-25.

Hou XH, Li XH (2015) Characteristics of land cover change in the Yellow River Estuary Nature Reserve from 1992 to 2010. Subtropical Plant Science, 44, 309-314. (in Chinese with English abstract) [侯学会, 李新华 (2015) 黄河三角 洲自然保护区1992 2010年土地覆被变化分析. 亚热带 植物科学, 44, 309-314.]

Huvenne VAI, Bett BJ, Masson DG, Bas TPL, Wheeler AJ (2016) Effectiveness of a deep-sea cold-water coral marine protected area, following eight years of fisheries closure. Biological Conservation, 200, 60-69.

IUCN (2012) Guidelines for Applying the IUCN Protected Area Management Categories to Marine Protected Areas http://www.iucn.org. (accessed on 2017-11-10)

Kaida N, Dang NA (2016) Tourists' perception of marine ecosystem conservation in the Nha Trang Bay Marine Protected Area, Vietnam. Tropics, 24, 187-194.

Kang M, Nakamura T, Hamano A (2011) A methodology for acoustic and geospatial analysis of diverse artificial-reef datasets. ICES Journal of Marine Science, 68, 2210-2221.

Kelleher G (1996) A global representative system of marine protected areas. Ocean and Coastal Management, 32, 123-126.

Li D (2017) Acoustic Observation and Evaluation of Benthic Habitat Evolution Under the Influence of Coastal Development. PhD dissertation, University of Chinese Academy of Sciences, Beijing. (in Chinese with English abstract) [李东 (2017) 近海开发影响下底栖生境演变的声学观测与评估 研究. 博士学位论文, 中国科学院大学, 北京.]

Li LH, Zhang HG, Shi AQ, Li DL (2013) Study on wetland landscape pattern change in the Ximen Island marine special protected area based on RS and GIS. Remote Sensing Technology and Application, 28, 129-136. (in Chinese with English abstract) [李利红, 张华国, 史爱琴, 厉冬玲 (2013) 基于RS/GIS的西门岛海洋特别保护区滩涂湿地景 观格局变化分析. 遥感技术与应用, 28, 129-136.]

Li XM, Zhang J, Ma Y, Ren GB (2017) Study on monitoring alien invasive species Spartina alterniflora using unmanned aerial vehicle hyperspectral remote sensing - a case study of the Yellow River Delta. Marine Sciences, 41(4), 98-107. (in Chinese with English abstract) [李晓敏, 张杰, 马毅, 任广 波 (2017) 基于无人机高光谱的外来入侵种互花米草遥 感监测方法研究——以黄河三角洲为研究区. 海洋科学, 41(4), 98-107.]
Lisa B, Daniel P (2016) Marine protection targets: An updated assessment of global progress. Oryx, 50, 27-35.

Ma KP (2016) Biodiversity monitoring relies on the integration of human observation and automatic collection of data with advanced equipment and facilities. Biodiversity Science, 24, 1201-1202. (in Chinese) [马克平 (2016) 生物多样性监测 依赖于地面人工观测与先进技术手段的有机结合. 生物 多样性, 24, 1201-1202.]

MBRS (2004) Manual for the Rapid Evaluation of Management Effectiveness in Marine Protected Areas of Mesoamerica. http://www.mbrs.doe.gov.bz/dbdocs/tech/Effective.pdf. (accessed on 2017-11-10)

Ouellette W, Getinet W (2016) Remote sensing for marine spatial planning and integrated coastal areas management: Achievements, challenges, opportunities and future prospects. Remote Sensing Applications Society and Environment, 4, 138-157.

Patterson TA, Sharples RJ, Raymond B, Welsford DC, Andrews-Goff V, Lea MA, Hindell M (2016) Foraging distribution overlap and marine reserve usage amongst sub-Antarctic predators inferred from a multi-species satellite tagging experiment. Ecological Indicators, 70, 531-544.

Pomeroy RS, Parks JE, Watson LM, Parks JE, Cid GA (2005) How is your MPA doing? A methodology for evaluating the management effectiveness of marine protected areas. Ocean and Coastal Management, 48, 485-502.

Quan J, Ouyang ZY, Xu WH, Miao H (2010) Comparison and applications of methodologies for management effectiveness assessment of protected areas. Biodiversity Science, 18, 90-99. (in Chinese with English abstract) [权佳, 欧阳志云, 徐卫华, 苗鸿 (2010) 自然保护区管理有效性评价方法 的比较与应用. 生物多样性, 18, 90-99.]

Reynolds SD, Norman BM, Beger M, Franklin CE, Dwyer RG (2017) Movement, distribution and marine reserve use by an endangered migratory giant. Diversity and Distributions, 23, 1268-1279.

Shao KZ, Lee H, Lin YC, Lai KQ (2014) A review of marine biodiversity information resources. Biodiversity Science, 22, 253-263. (in Chinese with English abstract) [邵广昭, 李瀚, 林永昌, 赖昆祺 (2014) 海洋生物多样性信息资源. 生物 多样性, 22, 253-263.]

Shaw JLA, Weyrich L, Cooper A (2016) Using environmental (e)DNA sequencing for aquatic biodiversity surveys: A beginner's guide. Marine and Freshwater Research, 68, 595-612.

Srivastava PK, Mehta A, Gupta M, Singh SK, Islam T (2015) Assessing impact of climate change on Mundra mangrove forest ecosystem, Gulf of Kutch, western coast of India: A synergistic evaluation using remote sensing. Theoretical and Applied Climatology, 120, 685-700.

Stolton S, Hockings M, Dudley N, MacKinnon K, Whitten T (2003) Reporting Progress in Protected Areas: A Site-Level Management Effectiveness Tracking Tool. http://documents. 
shihang.org/curated/zh/291981468171569997/pdf/32939a10ENGLIS1InProtectedAreasTool.pdf. (accessed on 2017 -11-10)

Thomas HL, Macsharry B, Morgan L, Kingston N, Moffitt R, Stanwell-Smith D, Wood L (2014) Evaluating official marine protected area coverage for Aichi Target 11: Appraising the data and methods that define our progress. Aquatic Conservation Marine \& Freshwater Ecosystems, 24(S2), 8-23.

Thomson JA, Borger L, Christianen MJA, Esteban N, Laloe JO, Hays GC (2017) Implications of location accuracy and data volume for home range estimation and fine-scale movement analysis: Comparing Argos and Fastloc-GPS tracking data. Marine Biology, 164, 204.

UNEP (2003) Training Manual: Training of Trainers Course in Marine Protected Area Management. UNEP and Coastal Zone Management Centre, Manila. http://cep.unep.org/calen dar/training-of-trainers-on-marine-protected-area-manageme nt-regional-course-1. (accessed on 2017-10-22)

Wang DG, Hu K, Ma PF, Wang MH, Liu X (2015) Land use and land cover change and their driving forces in the nature reserve of Shuangtai River Estuary in the past 30 years. Journal of Marine Sciences, 33(1), 51-61. (in Chinese with English abstract) [王丹桂, 胡克, 马鹏飞, 王铭晗, 刘霞 (2015) 双台河口自然保护区30a间土地利用/覆被变化及 其驱动力分析. 海洋学研究, 33(1), 51-61.]

Wang H (2017) China Nature Watch 2016. (in Chinese) [王吴 (2017) 中国自然观察 2016] http://chinanaturewatch.org. (accessed on 2017-12-25)

Wang W, Xin LJ, Du JH, Chen B, Liu FZ, Zhang LB, Li JS (2016) Evaluating conservation effectiveness of protected areas: Advances and new perspectives. Biodiversity Science, 24, 1177-1188. (in Chinese with English abstract) [王伟, 辛利娟, 杜金鸿, 陈冰, 刘方正, 张立博, 李俊生 (2016) 自然保护地保护成效评估: 进展与展望. 生物多样性, 24, 1177-1188.]

Wang ZF, Liu Q, Xu M, Li LB (2011) Health assessment of the ecosystem of the Liyashan Oyster Reef Special Marine Reserve of Haimen. Journal of Ecology and Rural Environment, 27(2), 21-27. [王在峰, 刘晴, 徐敏, 李露蓓 (2011) 海门市蚛岈山牡蚛礁海洋特别保护区生态系统健康评价, 生态与农村环境学报, 27(2), 21-27.]

Wenger AS, Williamson DH, da Silva ET, Ceccarelli DM, Browne NK, Petus C, Devlin MJ (2016) Effects of reduced water quality on coral reefs in and out of no-take marine reserves. Conservation Biology, 30, 142-153.

World Bank (2004) Score card to assess progress in achieving management effectiveness goals for marine protected areas. World Bank, Washington, DC. http://documents.worldbank. org/curated/en/101301468135588216/Score-card-to-assessprogress-in-achieving-management-effectiveness-goals-formarine-protected-areas. (accessed on 2017-11-10)

Wu W, Yan S, Feng R, Song D, Chen X (2017) Development of an environmental performance indicator framework to evaluate management effectiveness for Jiaozhou Bay Coastal Wetland Special Marine Protected Area, Qingdao, China. Ocean and Coastal Management, 142, 71-89.

Ye MB, Chen HL, Gu HX, Li PP (2015) Satellite tracking of migration for three captive-reared junvenile green turtles from Huidong National Sea Turtle Reserve. Sichuan Journal of Zoology, 34, 15-20. (in Chinese with English abstract) [叶明涁, 陈华灵, 古河祥, 李丕鹏 (2015) 人工培育幼年 绿海龟的卫星追踪试验. 四川动物, 34, 15-20.]

Ye YH, Peng SL, Hou YP, Yu YN, Liu KH, Chen BM, Ni GY, Song LY, Meng F, Li Y (2008) An analysis of development and distribution characteristics of marine nature reserves in China. Journal of Tropical Oceanography, 27(2), 70-75. (in Chinese with English abstract) [叶有华, 彭少麟, 侯玉平, 虞依娜, 刘可慧, 陈宝明, 倪广艳, 宋莉英, 孟帆, 李岩 (2008) 我国海洋自然保护区的发展和分布特征分析. 热 带海洋学报, 27(2), 70-75.]

Zeh DR, Heupel MR, Hamann M, Limpus CJ, Marsh H (2016) Quick Fix GPS technology highlights risk to dugongs moving between protected areas. Endangered Species Research, 30, 37-44.

Zeng JN (2013) Marine Protected Areas of China. China Ocean Press, Beijing. (in Chinese) [曾江宁 (2013) 中国海洋保护 区. 海洋出版社, 北京.]

Zeng JN, Chen QZ, Huang W, Du P, Yang H (2016) Reform of the marine ecological protection system in China: From marine protected areas to marine ecological redline regions. Acta Ecologica Sinica, 36, 1-10. (in Chinese with English abstract) [曾江宁, 陈全震, 黄伟, 杜萍, 杨辉 (2016) 中 国海洋生态保护制度的转型发展一一从海洋保护区走向 海洋生态红线区. 生态学报, 36, 1-10.]

Zhao ZZ, Liu RJ, Ma Y, Sun WF (2013) Remote sensing monitoring and analysis of coastline changes in the Meizhou Bay since the last 30 years. Coastal Engineering, 32(1), 19-27. (in Chinese with English abstract) [赵宗泽, 刘荣杰, 马毅, 孙伟富 (2013) 近30年来㳍洲湾海岸线变迁遥感监测与 分析. 海岸工程, 32(1), 19-27.]

(责任编委: 李俊生 责任编辑: 时意专) 